Man of many worlds

Paul Townsend

Quantum Theory of Gravity: Essays in Honor of the 60th Birthday of Bryce $S$. DeWitt.

Edited by Steven M. Christensen. Adam Hilger: 1984. Pp.483. £30, \$54.

ONE of the compliments paid to Bryce DeWitt in this collection of essays is that the strength of his work lies in its "unavoidability"; by this it is meant that "the development of science cannot escape it and, therefore, sooner or later it receives recognition". Overall the book is a good illustration of the remark.

The importance of much of DeWitt's pioneering work in quantum gravity, which is at the root of a number of areas of current research, is only now becoming apparent to many of us who entered the field at a later date. The first four essays in the book chart the course of some of these early ideas in frankly celebratory style, paying tribute to DeWitt's role in their formation or development. These contributions are the kind of idiosyncratic personal history that is naturally absent in journal articles and textbooks and which can be very instructive, even for the reader who is not familiar with the cast of characters. In particular I found the essay by Isham especially interesting as a concise bird's-eye view of the field.

The contributions that follow in the first third of the book take up the theme of quantum field theory in curved spacetime. Among them are accounts of some applications to cosmology and black hole physics, as well as a few that address conceptual problems of this approach. An example of the latter is Davies's provocatively titled essay "Particles do not exist".

The articles constituting the remaining two-thirds of the book grapple, for the most part, more directly with quantum gravity proper. There is no discernible thematic unity here because there is no consensus on the correct way to proceed. As a result, the essays range over a variety of approaches including Hamiltonian methods and the Wheeler-DeWitt equation, renormalization group methods, supergravity, and two- and three-dimensional spacetime analogues of quantum gravity, with several excursions into higher derivative modifications of general relativity.

There is only one contribution devoted to what is perhaps DeWitt's most fruitful creation, the "background field method", but it is a remarkably good one; this is the essay by Vilkovisky with the rather uninformative title "The Gospel according to

- Adam Hilger now have their own sales of fice in North America, serving the United States, Canada and Mexico. Orders should be sent to Adam Hilger Ltd, PO Box 230, Accord, MA 02018 , not to Heyden as previously.
DeWitt". A later article by Stelle reminds us of the power of this method in applications to supersymmetric field theories, supergravity in particular, although the latter subject is represented only briefly. Not represented at all is the "Kaluza-Klein" approach, currently very popular in its conjunction with supergravity, but neither of these topics has been influenced greatly by Bryce DeWitt. Finally, towards the end of the book, there is an essay by Smolin reminding us that DeWitt is one of the few physicists who have taken more than a passing interest in those quasiphilosophical problems that bedevil the interpretation of quantum mechanics; he is a well-known advocate of the bizarre "many worlds" interpretation.

\section{Unity in diversity?}

\section{Athel Cornish-Bowden}

\section{Biochemical Adaptation.}

By Peter W. Hochachka and George N.

\section{Somero.}

Princeton University Press: 1984. Pp.537. Hbk \$60, £60; pbk \$19.50, £15.

ALTHOUGH biologists have always been impressed by the diversity of life, biochemists have, on the contrary, emphasized its essential unity. All organisms use the same metabolites, arranged into the same series of metabolic reactions, catalysed by similar enzymes synthesized by the same kind of genetic apparatus. Biochemists have found that they can concentrate on a few organisms, and have done so to the extent that biologists have been known to complain that Escherichia coli is the only animal they know.

Nonetheless, life exists not only at $37^{\circ} \mathrm{C}$ at atmospheric pressure in the presence of water and oxygen, but also in many other habitats that might appear impossibly hostile, such as hot springs, frozen deserts and the ocean deeps. Even quite familiar animals can endure conditions that one might suppose to be lethal: the goldfish, for example, can survive in the complete absence of molecular oxygen for several days.

The pioneer in the field of biochemical diversity was Ernest Baldwin, whose little book An Introduction to Comparative Biochemistry (published by Cambridge University Press in 1970, but dating originally from 1937) still provides a stimulating entry into the subject. Much has been learned since his time, and Peter Hochachka and George Somero have written a much larger book, Biochemical Adaptation, with the aim of making biochemists more aware of the vast range of habitats in which life is possible and of showing how the diversity can nonetheless be rationalized in terms of a single biochemical theme.
On the whole I have the impression that the contributors have made a serious effort to write stimulating, readable essays, and this immediately puts the book in a category above most conference reports on quantum gravity. Also, discounting conference reports, I believe that this is the only collection of essays on quantum gravity extant, probably because no other figure of comparable stature has yet reached the age of 60 . While similar volumes will surely follow, this one is likely to remain a valuable source for those interested in the early years of quantum gravity.

Paul Townsend is in the Department of Applied Mathematics and Theoretical Physics at the University of Cambridge.

I believe the authors to have been successful in presenting the range of their subject. But I am far from happy with their rationalization, which seems to stretch theoretical chemistry to breaking point in many instances. The chapter on temperature adaptation gives prominence to entropy-enthalpy compensation, the idea that the enthalpies of activation of enzymes catalysing the same reaction in different organisms vary linearly with the corresponding entropies of activation. Some authors have apparently even found a mystic significance in the fact that the slopes of "compensation plots" are often close to $310 \mathrm{~K}$ (i.e. $37^{\circ} \mathrm{C}$ ). Yet nowhere is there any recognition of the fact that the numbers plotted can only be obtained by extrapolating to absolute zero a trend measured over perhaps $5^{\circ} \mathrm{C}$ to $40^{\circ} \mathrm{C}$, an extrapolation some eight times the range of measurement! Artificial correlation between slope and intercept is thus inevitable regardless of whether there is a real physical phenomenon.

Elsewhere in the same chapter, the authors speculate on possible reasons why such enzymes as lactate dehydrogenase may be more "efficient" in some organisms than in others. This may be a meaningless question how ever it is formulated; it is certainly meaningless when the "efficiencies" span a range as small as 0.54 to 1.00 , when they are defined in terms of the rate at substrate saturation and not at a physiological substrate concentration, and when they refer to a standard temperature that is unphysiological for some of the enzymes compared.

In the words of Theodosius Dobzhansky, "nothing makes sense in biology except in the light of evolution". Evolution makes only fleeting appearances in the book, but it might have been more convincing as a central theme than an appeal to physical chemistry which seems premature given our present state of knowledge.

Athel Cornish-Bowden is a Lecturer in the Department of Biochemistry at the University of Birmingham, UK. 